\title{
Medical Image De-Noising Schemes using Wavelet Transform with Fixed form Thresholding
}

\author{
Nadir Mustafa ${ }^{1}$ \\ ${ }^{1}$ School of Computer Science \&Technology, \\ UESTC, Chengdu, 611731, China
}

Saeed Ahmed Khan ${ }^{3}$

${ }^{3}$ Department of Electrical Engineering, Sukkur Institute of Business administration Sindh, Pakistan

\author{
Jiang Ping $\mathrm{Li}^{2}$ \\ ${ }^{2}$ School of Computer Science \& Technology, \\ UESTC, Chengdu, 611731, China
}

\author{
Mohaned Giess ${ }^{4}$ \\ ${ }^{4}$ School of Communication \& Information Engineering, \\ UESTC, Chengdu, 611731, China
}

\begin{abstract}
Medical Imaging is currently a hot area of bio-medical engineers, researchers and medical doctors as it is extensively used in diagnosing of human health and by health care institutes. The imaging equipment is the device, which is used for better image processing and highlighting the important features. These images are affected by random noise during acquisition, analyzing and transmission process. This condition results in the blurry image visible in low contrast. The Image De-noising System (IDs) is used as a tool for removing image noise and preserving important data. Image de-noising is one of the most interesting research areas among researchers of technology-giants and academic institutions. For Criminal Identification Systems (CIS) \& Magnetic Resonance Imaging (MRI), IDs is more beneficial in the field of medical imaging. This paper proposes an algorithm for de-noising medical images using different types of wavelet transform, such as Haar, Daubechies, Symlets and Bi-orthogonal. In this paper noise image quality has been evaluated using filter assessment parameters like Peak Signal to Noise Ratio (PSNR), Mean Square Error (MSE) and Variance, It has been observed to form the numerical results that, the presentation of proposed algorithm reduced the mean square error and achieved best value of peak signal to noise ratio (PSNR). In this paper, the wavelet based de-noising algorithm has been investigated on medical images along with threshold.
\end{abstract}

Keywords-Image De-noising System; GUI De-noised image; Code De-noised image; Wavelet transform; Soft and Hard Threshold

\section{INTRODUCTION}

Recently most of human-assistedcomputer applications rely on the use of digital image processing techniques, such as magnetic resonance imaging (MRI), criminal identification systems (CIS), agricultural and biological research (ABR). The term image de-noising is the best tool used in these applications, where it aims at remove the noise and retain important image features as much as possible. The use of medical imaging (MRI) in diagnosis has been greatly accepted for its non-sensitive features, low cost, the ability of constructing real-time image with improved property[1], [2].During image acquisition and transmission, it has been usually observed that random noise always occurs at another end. So this noise causes problems such as a blurred vision of images, which reduce the visuality of low-contrast articles.
Therefore, it is not easy for the medical doctors to examine the abnormalities in human in the invisible image. The process of removing noise is necessary in most medical imaging equipments for the purpose of enhancing miniatures that may be concealed in the data [3][4].

\section{WAVELET TRANSFORM}

This wavelet transform is alike to Windowed Fourier Transform (WFT), but themerit function is totally different. The main difference between the Window Fourier Transform and wavelet lies in the signal analysis; The WFT breaks down the signal into cosines and sines and, namely, the functions are restrained in Fourier space. On the contrary, functions that are utilized in the wavelet transform are confined in the real space and the Fourier space. Commonly, the Continuous Wavelet Transform (CWT) is containing different parameters which are derived from Fourier analysis transform and mother wavelet transform. The equation (1) describes the parameter $\gamma(s, \tau)$ is a wavelet coefficient with scale $s$ and time $\tau$, and the function $f(t)$ is define as the time series wherethe certain function is $\psi_{s, \tau}^{*}$ defines a complex conjugate of wavelet with scale and times, $\tau$.[5][6].

$$
\gamma(\mathrm{s}, \tau)=\int \mathrm{f}(\mathrm{t}) \psi_{\mathrm{s}, \tau}^{*}(\mathrm{t}) \mathrm{dt}
$$

Wavelets have been considered recently as a strong tool for de-noising image. The individual wavelet makes an image into a group of coefficients that compose a multi-scale model of the image. The distinct wavelet transform of signal expressed as $\mathrm{x}(\mathrm{n})$ is calculated by making it go through a low pass filter with impulse response $\mathrm{g}(\mathrm{n})$ as long as given an approximation coefficient. The signal is breaks down concurrently by the use of a high pass filter $\mathrm{h}(\mathrm{n})$, while gives details coefficients. These filters are named asQuadratic Mirror Filters. Because thehalf of frequencies of the signal is taken out, the sample of the filter outputs are reduced by equation (2)\&(3).

$$
\begin{aligned}
Y_{\text {low }}[k] & =\sum_{n} x[n] \cdot g[2 k-n] \\
Y_{\text {high }}[k] & =\sum_{n} x[n] \cdot g[2 k-n]
\end{aligned}
$$


Image is a 2-dimentional signal, and we use $\mathrm{x}(\mathrm{N}, \mathrm{M})$ to represent it. Firstly each row is filtrated and then down-sampled to get two images represented by $(\mathrm{N}, \mathrm{M} / 2)$, secondly every column is filtrated and down-sampled toget four sub bands named as HH, HL, LH and LL Therefore, in case of two dimensions, one 2-D scaling function and three 2-D wavelet functionsare generated.

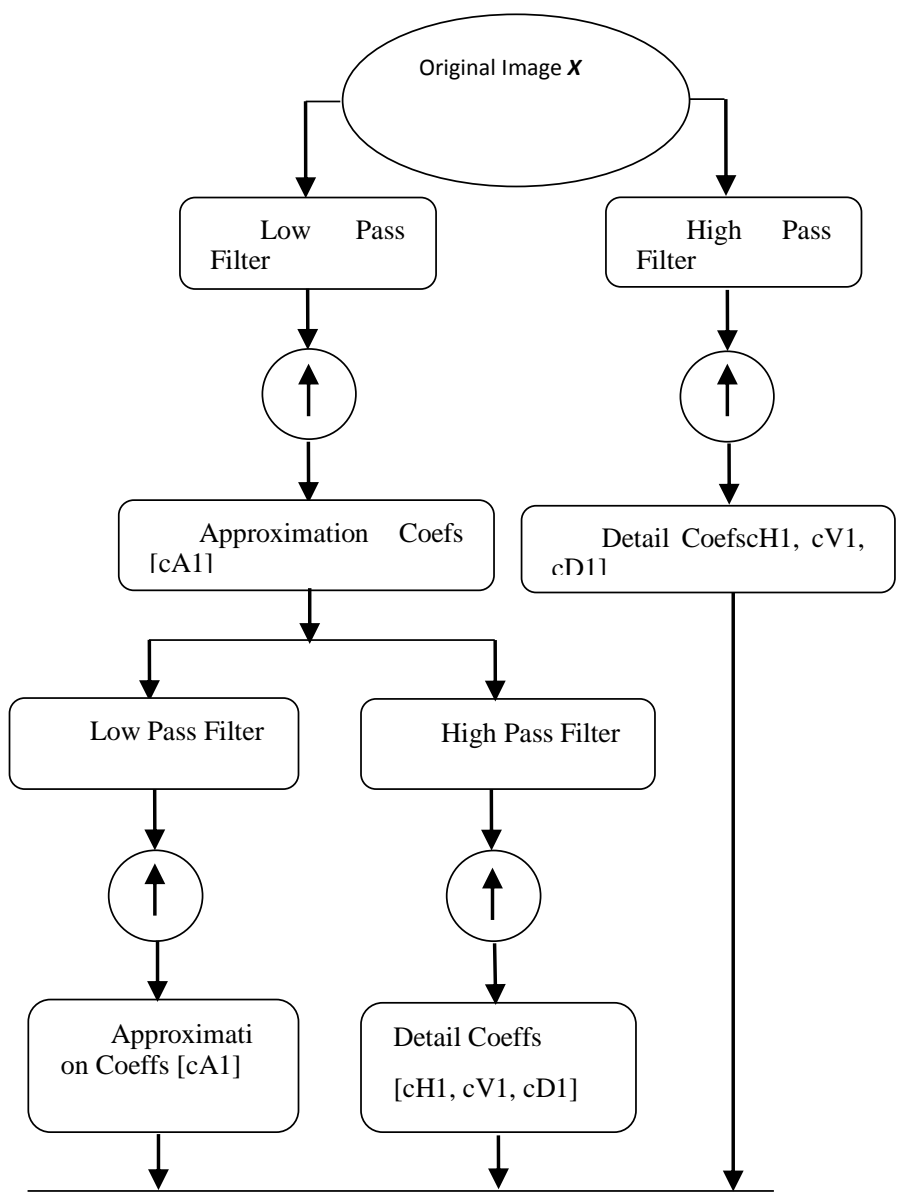

Fig. 1. The 2D discrete wavelet decomposition

The variable CA1, CD1, CH1 and CV1 stand for approximation coefficient, diagonal detail coefficient, horizontal detail coefficient and vertical detail coefficient.

At level two approximation sub-band LL is then decomposed into four components, the performance can be progressed all the same for anotherthree levels. LL has strength concentration for low pass and $\mathrm{HH}$ sub-band for high-frequency constituents. Rebuilding can be performed by IDWT (Inverse Discrete Wavelet Transform) to obtain the de-noised image [7] [8].

The process of 2D discrete wavelet decomposition has been depicted in figure.1, which describes the main steps for de-noising. The process starts from image decomposition, up-sampling and down-sampling until the reconstruction of four sub band coefficients are obtained for original image [9][10].

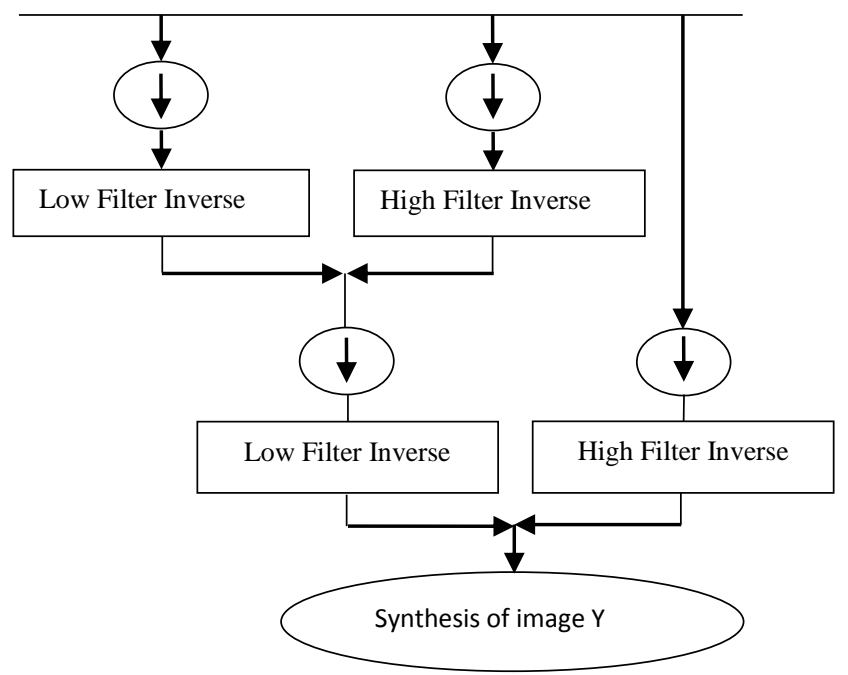

Fig. 2. The 2D discrete wavelet reconstruction

Here, figure. 2 illustrates; brain image reconstruction from three-level decomposition. We can see the wavelet decomposition process can be seen byconsecutive approximations being decomposed successfully. In figure three the original medical image shows decomposition into many elements with lower-resolution.

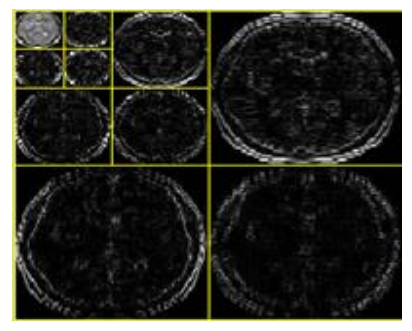

Fig. 3. Wavelet three-level decomposition of brain image

\section{PROPOSED ALGORTHIM FOR DE-NOISING}

The purpose of this paper is the de-noising of medical image of the brain usingdifferent types of wavelets, such as Haar, db10, sym3 and bior3.7 wavelet. Our contribution in this paper is that good results are obtained when applying fixed form threshold in terms of soft and hard threshold algorithm. To evaluate the proposed algorithm, several parameters are used such as Peak Signal to Noise Ratio (PSNR), Mean Square Error (MSE) and Variance. Numerical results show the validity of proposed algorithm. The mean square error is reduced, while a peak signal to noise ratio (PSNR) is achieved.

\section{A. Image De-noising Algorithm}

There are three steps of de-noising proceduredescribed as follows:

Wavelet decomposition level Pick a level (level-3). Calculate the wavelet decomposition of the noisy image at level 3. The wavelet produces all the coefficients, from the wavelet analysis process. 
Threshold detail coefficients:a threshold is chosen for level3 and softthresholdingisapplied to the detail coefficients. If the wavelet coefficients are larger than the threshold value, those coefficients are leftunaltered. If they are small than threshold, they are restrained.

Reconstruct wavelet coefficients based on level 3 of wavelet transform. Then, transformdetailed coefficients from level 3 to level 1.

\section{B. ThresholdingParameter}

In this part parameters are formulated andused for de-noising.

\section{1) Noise variance}

Apply a fixed form thresholding algorithm to the wavelet coefficients. In fixed form, the noise variance is calculated using the median of absolute deviation of the transform coefficient of all three levels; the (MAD) is given by equation (4).

$$
\sigma_{n}^{2}=\frac{\operatorname{median}\left(a b s\left(x_{i j}\right)\right)^{2}}{0.6745}
$$

\section{2) Threshold Parameter}

The threshold $\left(T_{h}\right)$ is a threshold parameter applied to wavelet coefficients of a noisy image. Where $M$ is number of pixels in theimage, and $S$ is the noise variance and the threshold is given by equation (5).

$$
T h=\sigma \sqrt{2 \log M}
$$

Hard thresholding is a keep or kill the wavelet coefficients compared with threshold parameter. The threshold is deducted from any coefficient that is larger than the threshold. This process makes the time series move toward zero.

\section{Evaluation Parameters}

In this partevaluation parameters are discussed.

\section{1) Mean Square Error (MSE)}

The MSE estimate the quality alteration between the GUI de-noised image $(\mathrm{X})$ and code demised image $(\mathrm{Y})$, the average of the squared image is given in equation (6).

$$
M S E=\frac{1}{m n} \sum_{i=0}^{m-1} \sum_{j=0}^{n-1}[Y(i, j)-X(i, j)]^{2}
$$

2) Peak Signal to Noise Ratio (PSNR)

The PSNR represents the size of the error in relation to the peak value of the signal rather than the size of the error in relation to the average squared value of the signal. It is computed with the size of the error in relation to the average squared value of the signal. PSNR is greater for a better-transformed image and smaller for a poorly transformed image. PNSR calculates image fidelity, i.e., intimately the transformed image looks like the initial image, the PSNR exhibited in equation (7).

$P S N R=10 \log _{10}\left[\frac{S^{2}}{m s e}\right]$

\section{RESEARCH METHODOLOGY}

The experiments in this paper have been conducted on two medical images; of Brain with different size. The first image is a brain medical image with size [204x204], the second image is a brain medical image with size [150x150]; Different types of wavelet transform have been applied respectively (haar, $\mathrm{db} 10$, sym3, and bior3.7) for these two images to generate de-noised image. After applying wavelet, (CA) approximation and (CD) details coefficient at three levels of decomposition process have been generated. These coefficients represented in vector [C,S] such as [CD1, CD2, CA3, CD3]. After each level consists of horizontal, vertical and diagonal coefficients, de nosing image is achieved. As there are many threshold levels but in this paper, fixed form soft threshold for three levels of decomposition process have been selected because it will give best threshold value. Here un-scaled white noise is added to the original image to generate a de-noising image in GUI (Graphical User Interface). At the first stage, the original image is compared with the GUI de-noising image. for the same scheme, MATLAB codesare written to compare the original image using hard threshold with the image de-noising generated code. At the later stage the GUI de-noising image is compared with the image de-noising generated code along with MSE and PSNR parameter.

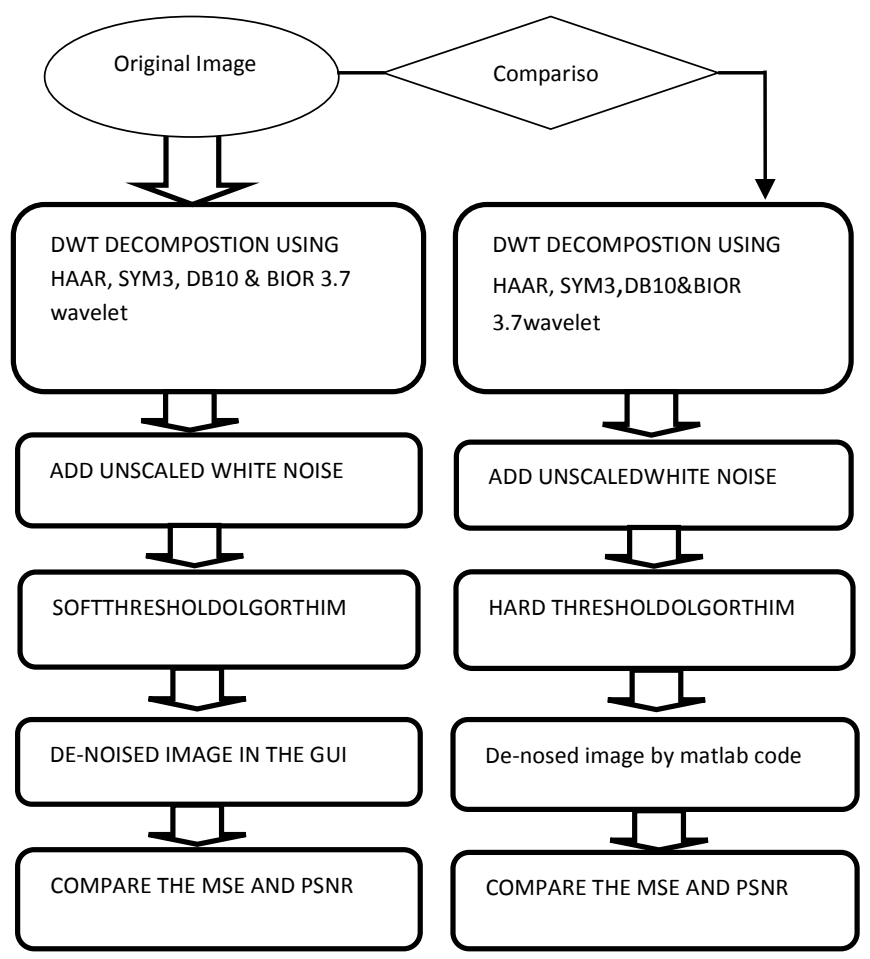

Fig. 4. De Nosing Comparison Algorithm Model

\section{RESULTS AND DISCUSSION}

Here Fig.5 illustrates the initialmedical image of brain image. Fig 6\&7 depicts the de-noising images generated in GUI and MATLAB Code. 


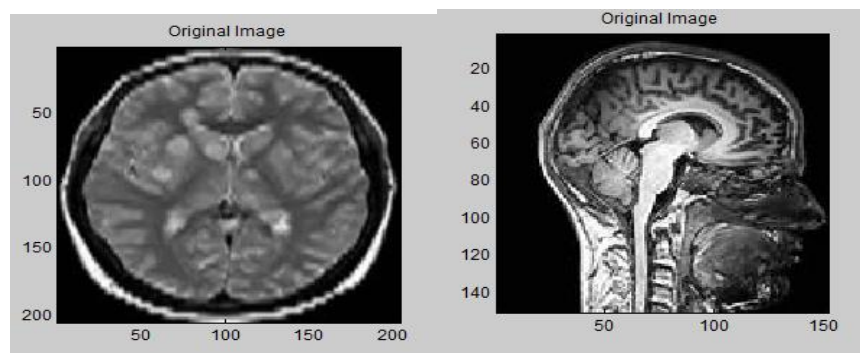

Fig. 5. The original of two brain images

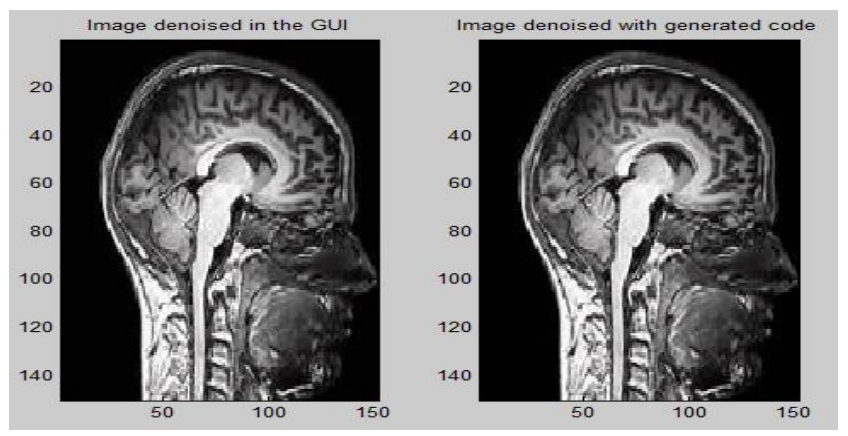

Fig. 6. The De-noised of first brain image

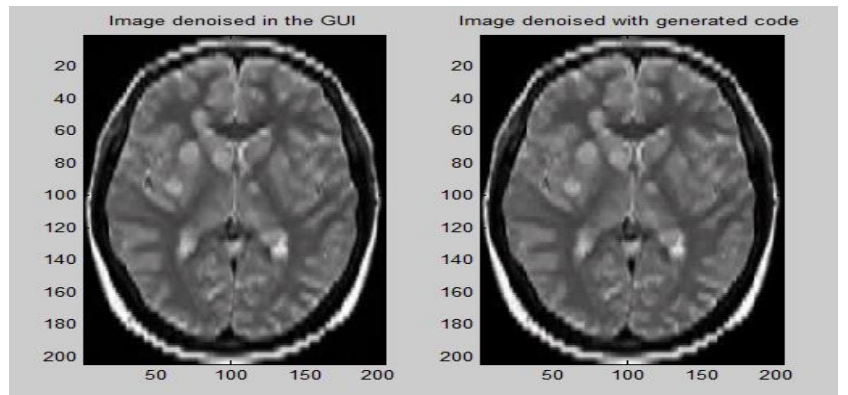

Fig. 7. The De-noised second brain image

The Performance evaluation of de-noising image can be observed in the tables $1 \& 2$. From the relationship of the peak signal and mean squareto the noise ratio, here it can be observed from Table $1 \& 2$ that the MSE measurements in the GUI generated de-noising image are smaller than the measurements in the code generated de-noising image in all wavelet families. It reveals that the mean square error of the initialimage in GUI is less than the hard threshold generated code of the original image. That is because of the image size.

TABle I. Performance Evaluation of Gui MEthod For DifFerent THRESHOLDING IN TERM OF MSE, PSNR FOR TwO DIFFERENT BRAIN IMAGES

\begin{tabular}{|r|l|l|l|l|l|}
\hline \multirow{2}{*}{$\begin{array}{l}\text { Various } \\
\text { image }\end{array}$} & \multirow{2}{*}{$\begin{array}{l}\text { Wavelet } \\
\text { package }\end{array}$} & \multicolumn{3}{|l|}{$\begin{array}{l}\text { Soft Threshold } \\
\text { Method }\end{array}$} & \multicolumn{2}{l|}{$\begin{array}{l}\text { Hard Threshold } \\
\text { Method }\end{array}$} \\
\cline { 3 - 6 } & & MSE & PSNR & MSE & PSNR \\
\hline $\begin{array}{l}\text { Brain } \\
\text { image } \\
\text { with size } \\
(150 \times 150)\end{array}$ & haar & 0.8436 & 48.2026 & 3.4461 & 41.7699 \\
\cline { 2 - 6 } & db10 & 0.8697 & 48.2200 & 3.2437 & 41.6534 \\
\cline { 2 - 6 } & sym3 & 0.7360 & 48.2309 & 3.2600 & 41.8367 \\
\hline bior3.7 & 0.6589 & 48.2433 & 2.6374 & 41.9085 \\
\hline Brain & haar & 0.8280 & 48.1395 & 4.3343 & 38.9221 \\
\hline
\end{tabular}

\begin{tabular}{|c|c|c|c|c|c|}
\hline \multirow{3}{*}{$\begin{array}{l}\text { image } \\
\text { with size } \\
(200 \times 200)\end{array}$} & $\begin{array}{ll} & \mathrm{db} 1 \\
0 & \end{array}$ & $595^{0.8}$ & $526^{48.1}$ & $580^{5.5}$ & $631^{39.9}$ \\
\hline & sym3 & 0.7062 & 48.1809 & 4.8423 & 40.2849 \\
\hline & bior3.7 & 0.6612 & 48.2085 & 3.4412 & 42.2548 \\
\hline
\end{tabular}

TABLE II. Performance EVAluation of Matlab Code Method For DIFFERENTTHRESHOLDING IN TERM OF MSE, PSNR FOR TwO DIFFERENT BRAIN IMAGES

\begin{tabular}{|l|l|l|l|l|l|}
\hline \multirow{2}{*}{$\begin{array}{l}\text { Various } \\
\text { Images }\end{array}$} & \multirow{2}{*}{$\begin{array}{l}\text { Wavelet } \\
\text { package }\end{array}$} & \multicolumn{2}{l}{$\begin{array}{l}\text { Soft Threshold } \\
\text { Method }\end{array}$} & \multicolumn{2}{l|}{$\begin{array}{l}\text { Hard Threshold } \\
\text { Method }\end{array}$} \\
\cline { 2 - 6 } & & MSE & PSNR & MSE & PSNR \\
\hline \multirow{2}{*}{$\begin{array}{l}\text { Brain } \\
\text { image } \\
\text { with size } \\
(150 \times 150)\end{array}$} & haar & 0.9080 & 48.1395 & 7.3343 & 38.9221 \\
\cline { 2 - 6 } & db10 & 0.9395 & 48.3085 & 4.5580 & 39.9631 \\
\cline { 2 - 6 } & bym3 & 0.8962 & 48.3709 & 5.8423 & 42.2849 \\
\hline \multirow{2}{*}{$\begin{array}{l}\text { Brain } \\
\text { image } \\
\text { with size } \\
(200 \times 200)\end{array}$} & Haar & 0.7212 & 48.6485 & 2.4412 & 44.2548 \\
\cline { 2 - 6 } & db10 & 0.9312 & 47.2095 & 9.4413 & 38.3264 \\
\cline { 2 - 6 } & bior3.7 & 0.7551 & 47.2526 & 7.2052 & 40.5231 \\
\hline
\end{tabular}

Fig. $8 \& 9$ illustrates the relationship of MSE \& PSNR of four wavelet families for brain de-noising medical image. Here it can be observed that bior3.7 wavelet has better results than the other wavelet families used in this paper for image de-noising.

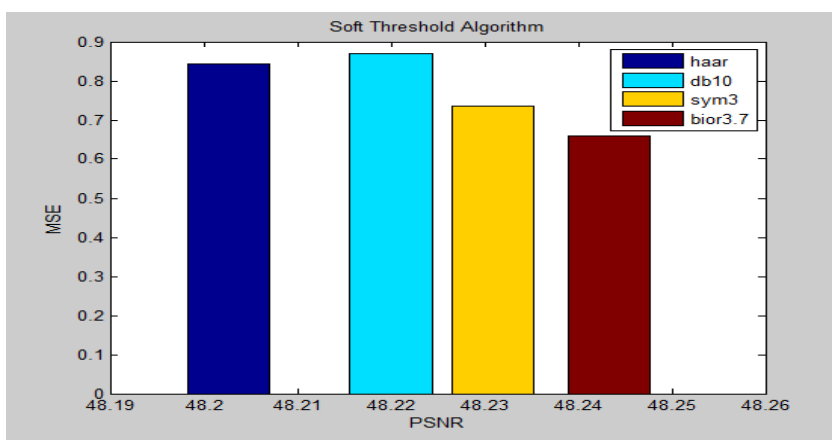

Fig. 8. The histogram of GUI method using Soft Threshold Algorithm for Brain de-noising image

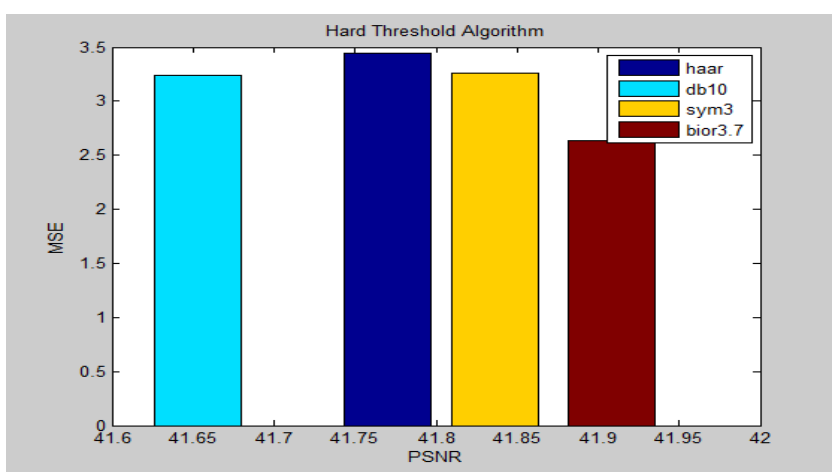

Fig. 9. The histogram of GUI method using Hard Threshold Algorithm for Brain de-noising image 
Fig. $10 \& 11$ illustrates the relationship of MSE \& PSNR of four wavelet families for the brain de-noising medical image. Here it can be observed that bior3.7 wavelet has better results than the other wavelet families used in this paper for image de-noising.

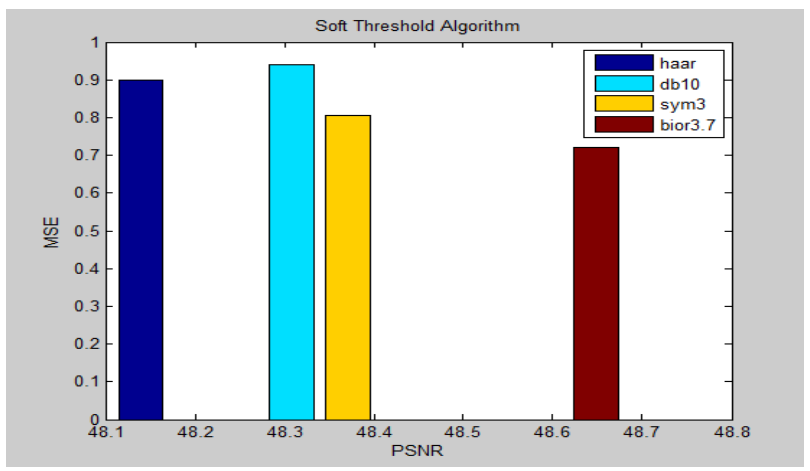

Fig. 10. The histogram of MATLAB code method using Hard Threshold Algorithm for brain de-noising image

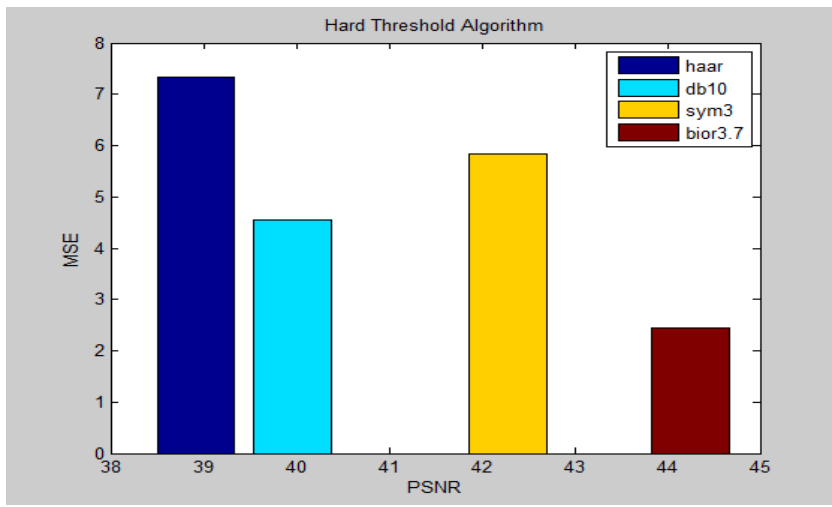

Fig. 11. MATLAB code method using Hard Threshold Algorithm for brainde-noising image

Fig. 12\& 13 illustrates the relationship of MSE \& PSNR of four wavelet families for the brain de-noising medical image. Here it can be observed that bior3.7 wavelet has better results than the other wavelet families used in this paper for image de-noising.

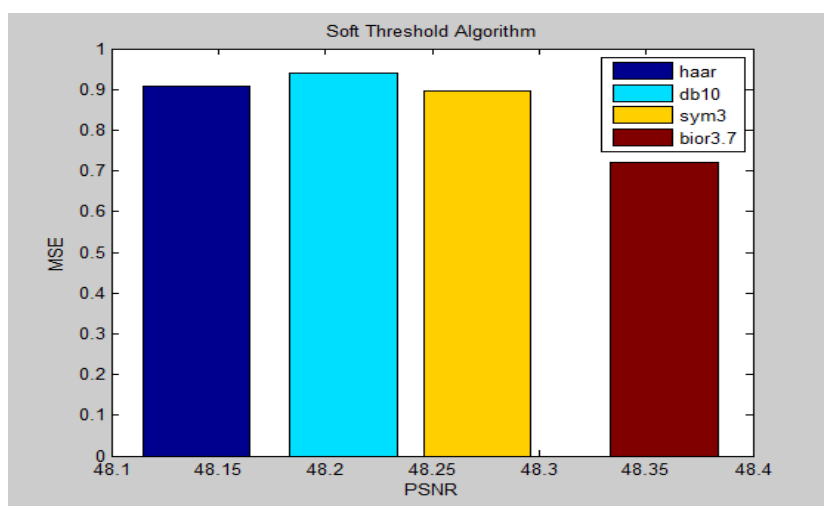

Fig. 12. The histogram of GUI method using Soft Threshold Algorithm for brain de-noising image

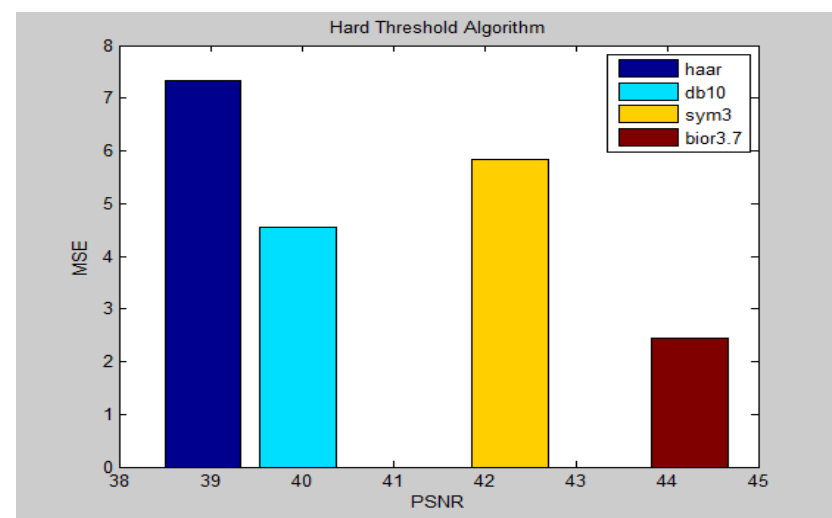

Fig. 13. The histogram of GUI method using Hard Threshold Algorithm for brain de-noising image

Fig. 14\& 15 illustrates the relationship of MSE \& PSNR of four wavelet families for the de-noising medical image. Here it can be observed that bior3.7 wavelet has better results than the other wavelet families used in this paper for image de-noising.

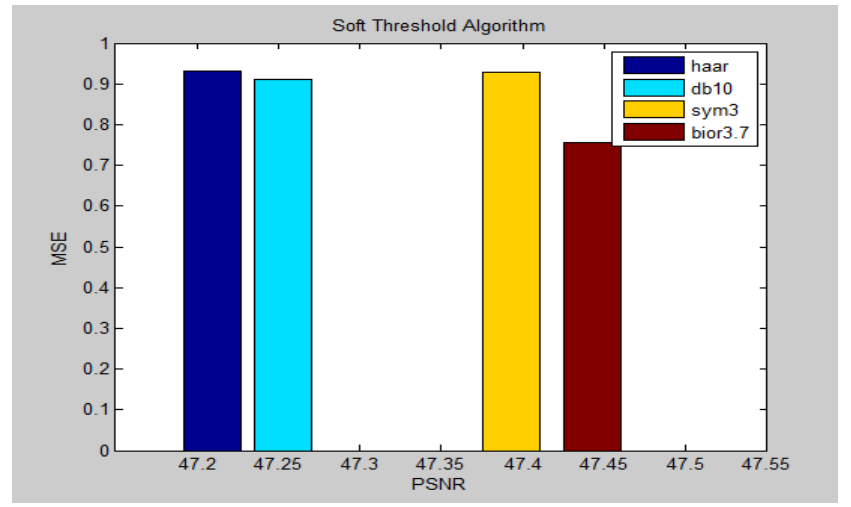

Fig. 14. The histogram of MATLAB code method using Hard Threshold Algorithm for Brain de-noising image

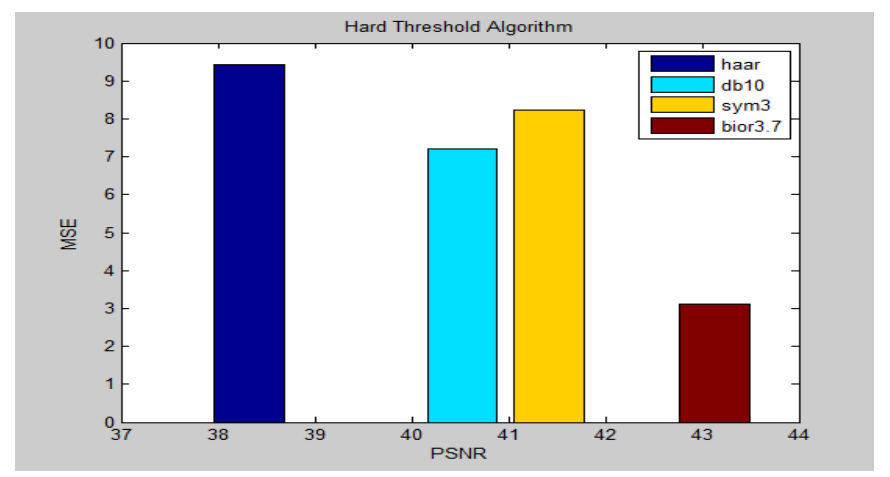

Fig. 15. The histogram of MATLAB code method using Hard Threshold Algorithm for Brain de-noising image

\section{CONCLUSIONS}

In all images, noise is the main problem, and one has to nip this problem in the bud for better results. 
De-noising is very crucial especially in medical science. In this paper, removing the un-scaled white noise added to original medical images has been presented. The new algorithm has been proposed for De-noising brain medical images. The proposed new algorithm on the basis of the wavelet transform is observed to be a more competent method in image de-noising especially forremoving un-scaled white noise. Qualitative and quantitative analysis results reveal that the proposed algorithm reduces the mean square error (MSE) of different images with different sizes using different wavelet families for hard and soft threshold. Experimentsrepresent that, the bi-orthogonal wavelet is a more efficient method than other wavelet families discussed in this paper, such as Haar, Daubechies, and Symlets because it gave better results with mean square error (MSE) in soft and hard threshold. Efficient de-noising values in a soft threshold algorithm are generated in GUI. Because of difference in image sizes, hard threshold algorithm generated code values were observed as larger in case of the brain medical image. Results reveal that bi-orthogonal wavelet shows the best results with parameter MSE and PSNR. At the later stages one can work precisely on MSE and PSNR measurements for both soft and hard thresholds for getting de-noised medical images.

\section{ACKNOWLEDGEMENT}

This paper was supported by the National Natural Science Foundation of China (Grant No: 61370073), and the National High Technology Research and Development Program of China (Grant No: 2007AA01z423).

\section{REFERENCES}

[1] A. Bultheel: Bull. Belg. Math. Soc.: (1995) 2

[2] S.Satheesh, Dr.KVSVR Prasad, "Medical image de-noising using adaptive threshold based on contourlet transform" , An International Journal ( ACIJ), Vol.2, No.2, March 2011.

[3] S. G. Chang, B. Yu, M. Vetterli: IEEE Trans. Image Processing, (2000) 9 p. 1532

[4] S. G. Chang, B. Yu, M. Vetterli: IEEE Trans. Image Processing, (2000) 9 p. 1522

[5] Sivakumar .R and Nedumaran .D, "Implementation of Wavelet Filters for Speckle Noise Reduction in Ultrasound Medical Images: A Comparative Study" International Conference on Signals, Systems and Communication, December 21-23, 2009.

[6] AsmaaAbassAjwad, “ Noise Reduction of Ultrasound Image Using Wiener filtering and Haar Wavelet Transform Techniques" Diyala Journal of Medicine, Vol. 2, Issue 1, April 2012.

[7] Dhruv Kumar, MaitreyeeDutta, ParveenLehane, " A Comparative Analysis of Different Wavelets for Enhancing Medical Ultrasound Images", International Journal

[8] Eslami, R. and Radha. H., "Translation-invariant contourlet transform and its application to image denoising," IEEE Transactions on Image Processing, Vol. 15, No.11, pp. 3362-3374, 2006

[9] M. N. Do and M. Vetterli, "Pyramidal directional filter banks and curvelets," Proc. IEEE Int. Conf. on Image Proc., vol. 3, 2001, pp. 158-161.

[10] M. N. Do and M. Vetterli, "Contourlets: a Directional Multiresolution image representation," Proceedings of 2002 IEEE International Conference on Image Processing, vol. 1, 2002, pp 357-360. 